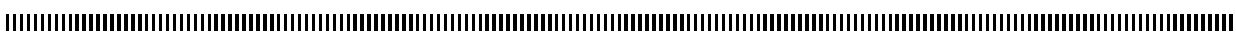

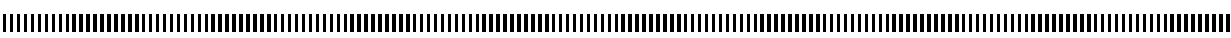
| |

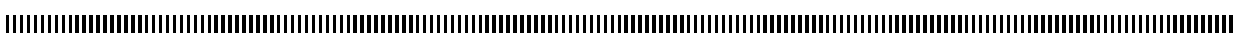

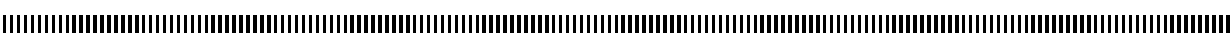
| | | |

\title{
A posteriori error estimates for non conforming approximation of quasi Stokes problem
}

\author{
B. Achchab ${ }^{a}$, A. Agouzal ${ }^{b}$, and K. Bouihat ${ }^{a}$, \\ ${ }^{a}$ Univ. Hassan $1^{e r}$, LAMSAD, Ecole Supérieure de Technologie de Berrechid et LM ${ }^{2} \mathrm{CE}$ \\ Faculté des Sciences Economiques, Juridiques et Sociales Settat, B.P 218 Berrechid, Maroc. \\ Corresponding author: achchab@estb.ac.ma; \\ khalidbouihat@yahoo.fr \\ ${ }^{b}$ Univ. Lyon; CNRS; Université Lyon 1; Institut Camille Jordan, F-69622 Villeurbanne cedex, \\ France. \\ agouzal@univ-lyon1.fr
}

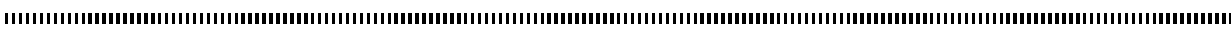

ABSTRACT. We derive and analyze an a posteriori error estimator for nonconforming finite element approximation for the quasi-Stokes problem, which is based on the solution of local problem on stars with low cost computation, this indicator is equivalent to the energy error norm up to data oscillation, neither saturation assumption nor comparison with residual estimator are made.

RÉSUMÉ. Nous dérivons et analysons un estimateur d'erreur a posteriori pour les approximations par éléments finis non conformes du problème de quasi-Stokes, il est basé sur la résolution de problèmes locaux à faible coût de calcul. L'estimateur est équivalent à l'erreur en norme d'énergie augmentée d'oscillations des données, sans hypothèse de saturation ni de comparaison avec l'estimateur par résidu.

KEYWORDS : A posteriori error estimator, nonconforming finite elements method, quasi-Stokes problem.

AMS subject classification: 65D05, 65D15, 65N50.

MOTS-CLÉS : Estimateur d'erreur a posteriori, Méthode des éléments finis non conformes, problème de quasi-Stokes.

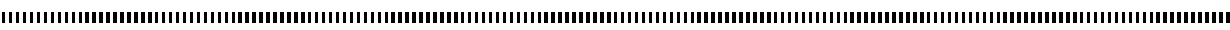

Received, December 12, 2011, ARIMA Journal, vol. 16 (2013), pp. 47-66.

Revised, November 9, 2012,

Accepted, October 14, 2013 


\section{Introduction}

The need for accurate solutions of large scale problems in computational fluid dynamics has made the use of adaptive, automatic re-meshing very attractive for nonconforming finite element approximations to solution of partial differential equation. A posteriori error estimate was introduced in order to provide an information about the local and global quality of the computed finite element solution. One of the most popular a posteriori error estimators is the hierarchical estimator introduced by Bank et al. in [8] for symmetric elliptic problems and generalized after by Achchab et al. in [2] and Hoppe et al. in [14] for conforming and nonconforming mixed finite elements. An abstract setting is developed in $([1,4,5,6,15])$.

In this paper we developed and analyzed an error estimate for the quasi-Stokes problem which is based on the solution of a local quasi-Stokes problem in star, this estimate is shown to both a global upper and lower bound of the discretization error ([7],[8],[13], [15]).

The advantage of using the solution of the local problem on star is control error without influence on the regions near the singularity which introduces less degree of freedom and the optimality of cost calculation ([3],[9],[10], [15]).

We test this estimate on several classical problems and demonstrate its efficiency in grid adaptation. The quasi Stokes problem modeling the fluid flows, and flows in porous media.

\section{Setting of the problem}

We consider the quasi Stokes problem :

$$
\left\{\begin{array}{c}
\text { Find }(u, p) \text { such that } \\
-\Delta u+\sigma u+\nabla p=f \text { in } \Omega, \\
\text { divu }=0 \text { in } \Omega, \\
u=0 \text { on }=\Gamma=\partial \Omega,
\end{array}\right.
$$

where $f \in\left(L^{2}(\Omega)\right)^{d}$. We assume that $\sigma \in L^{\infty}(\Omega)$ and $\Omega \subset \mathbf{R}^{d}, d=2,3$, is a simply connected polygonal domain, $u$ is the velocity, $p$ is the pressure. Let $\left(\mathcal{T}_{h}\right)_{h}$ be a family of conforming shape-regular triangulation of $\Omega$ by d-simplex. We denote by $E_{I}$ the set of interior edges (faces), and by $E_{f}$ the set of all edges (faces) included in $\Gamma$.

We denote by $\left\{x_{i}\right\}_{i \in \mathcal{N}}$ the set of all nodes of the triangulation $\mathcal{T}_{h}$. For each $i \in \mathcal{N}$, $\phi_{i}$ denotes the canonical continuous piecewise linear basis function associated to $x_{i}$. The star $\omega_{i}$ is the interior relative to $\Omega$ of the support of $\phi_{i}$, and $h_{i}$ is the maximal size of the elements constituting $\omega_{i}$. Finally, $\Gamma_{i}$ denotes the union of the sides touching $x_{i}$ that are contained in $\Omega$, and $\bar{\Gamma}_{i}$ the union of the sides touching $x_{i}$ that are contained in $\bar{\Omega}$.

Let $V_{h}(\Omega)$ be the lowest order non-conforming Crouzeix-Raviart finite element space defined by 
$V_{h}(\Omega)=\left\{v_{h} \in L^{2}(\Omega) ; \forall T \in \mathcal{T}_{h}, v_{h \mid T} \in P_{1}(T), \forall E \in E_{I}, \int_{E}\left[v_{h}\right]_{E} d \sigma=0\right.$ and $\left.\forall E \in E_{f}, \int_{E} v_{h} d \sigma=0\right\}$.

and

$$
Q_{h}=\left\{q_{h} \in L_{0}^{2}(\Omega), q_{h \mid T} \in P_{0}(T), \forall T \in \omega_{i} \text { and } \omega_{i} \in \mathcal{T}_{h}\right\} .
$$

where $[\cdot]_{E}$ denotes the jump of the argument across $E$.

For each star $\omega_{i}, i \in \mathcal{N}$, if $x_{i}$ is an interior node, we introduce the space defined by

$$
V\left(\omega_{i}\right)=\left\{v \in\left(H_{l o c}^{1}\left(\omega_{i}\right)\right)^{d}: \int_{\omega_{i}} v \phi_{i} d x=0 \text { and } \int_{\omega_{i}}|\nabla v|^{2} \phi_{i}<\infty\right\},
$$

and if $x_{i}$ is a boundary node, by

$$
V\left(\omega_{i}\right)=\left\{v \in\left(H_{l o c}^{1}\left(\omega_{i}\right)\right)^{d}: v=0 \text { on } \partial \omega_{i} \cap \Gamma \text { and } \int_{\omega_{i}}|\nabla v|^{2} \phi_{i}<\infty\right\} .
$$

There exists a constant $C$, only depending on the minimum angle of the triangulation but independent of the star being considered, such that (see Prop. 2.4 of [15]) :

$$
\forall v \in V\left(\omega_{i}\right) ; \quad\|v\|_{0, \omega_{i}} \leq C h_{i}\left(\int_{\omega_{i}}|\nabla v|^{2} \phi_{i} d x\right)^{1 / 2} .
$$

We define the finite dimensional local space $\mathcal{P}_{0}^{2}\left(\omega_{i}\right)$ as follows,

Definition 1 For $i \in \mathcal{N}$, let $\mathcal{P}^{2}\left(\omega_{i}\right)$ denote the space of continuous piecewise quadratic functions on star $\omega_{i}$ that vanish on $\partial \omega_{i}$. The spaces $\mathcal{P}_{0}^{2}\left(\omega_{i}\right)$ are defined by $\mathcal{P}_{0}^{2}\left(\omega_{i}\right)=\mathcal{P}^{2}\left(\omega_{i}\right) \cap V\left(\omega_{i}\right)$.

We consider the approximate solution $\left(u_{h}^{N C}, p_{h}\right) \in\left(V_{h}(\Omega)\right)^{d} \times Q_{h}$ defined by:

$$
\left\{\begin{array}{l}
\forall v_{h} \in\left(V_{h}(\Omega)\right)^{d}, \\
\sum_{T \in \mathcal{T}_{h}}\left\{\int_{T} \nabla u_{h}^{N C}: \nabla v_{h} d x+\int_{T} \sigma u_{h}^{N C} \cdot v_{h} d x-\int_{T} p_{h} \operatorname{div} v_{h} d x\right\}=\int_{\Omega} f \cdot v_{h} d x \\
\forall q_{h} \in Q_{h}, \quad \sum_{T \in \mathcal{T}_{h}} \int_{T} q_{h} \operatorname{div} u_{h}^{N C} d x=0
\end{array}\right.
$$

Note that the second equation means that for every $T \in \mathcal{T}_{h}, \operatorname{div}\left(\left(u_{h}^{N C}\right)_{\mid T}\right)=0$.

Let $v_{h} \in\left(V_{h}(\Omega)\right)^{d}$ be fixed. We define $\nabla_{h} v_{h}$ and $\operatorname{div}_{h} v_{h}$ by:

$$
\forall T \in \mathcal{T}_{h} ; \quad \nabla_{h} v_{h}=\nabla v_{h} \quad \text { on } T
$$

and

$$
\forall T \in \mathcal{T}_{h} ; \quad \operatorname{div}_{h} v_{h}=\operatorname{div} v_{h} \text { on } T .
$$


For each $i \in \mathcal{N}$, we consider the local problems :

$\left(S P_{i}\right)\left\{\begin{array}{r}\text { Find } \eta_{i} \in\left(\mathcal{P}_{0}^{2}\left(\omega_{i}\right)\right)^{d}, \text { such that } \forall \mu_{i} \in\left(\mathcal{P}_{0}^{2}\left(\omega_{i}\right)\right)^{d}, \\ \int_{\omega_{i}}\left(\nabla \eta_{i}: \nabla \mu_{i}\right) \phi_{i} d x=\int_{\omega_{i}} \nabla_{h} u_{h}^{N C}: \nabla\left(\mu_{i} \phi_{i}\right) d x+\int_{\omega_{i}} \sigma u_{h}^{N C} \cdot \mu_{i} \phi_{i} d x \\ -\int_{\omega_{i}} p_{h} \operatorname{div}\left(\mu_{i} \phi_{i}\right) d x-\int_{\omega_{i}}\left(f . \mu_{i}\right) \phi_{i} d x .\end{array}\right.$

It is obvious that these local problems admit unique solutions.

We introduce for all $i \in \mathcal{N}$ the three indicators,

$$
\begin{array}{r}
\eta_{1, i}\left(u_{h}^{N C}, p_{h}\right)=\left(\sum_{T \in \omega_{i}}\left\|\operatorname{div} u_{h}^{N C} \phi_{i}^{\frac{1}{2}}\right\|_{0, \omega_{i}}\right)^{\frac{1}{2}}, \\
\eta_{2, i}\left(u_{h}^{N C}, p_{h}\right)=\left(\int_{\omega_{i}}\left|\nabla \eta_{i}\right|^{2} \phi_{i} d x\right)^{\frac{1}{2}},
\end{array}
$$

and

$$
\eta_{3}^{2}\left(u_{h}^{N C}, p_{h}\right)=\sum_{E \in E_{I}} h^{-1}\left\|\left[u_{h}^{N C}\right]\right\|_{0, E},
$$

and set the problem data oscillation,

$$
\operatorname{osc}(f)=\left(\sum_{i \in \mathcal{N}} h_{i}^{2}\left\|\left(f-f_{i}\right) \phi_{i}^{\frac{1}{2}}\right\|_{0, \omega_{i}}^{2}\right)^{\frac{1}{2}} .
$$

where $f_{i}=\frac{\int_{\omega_{i}}\left(f-\sigma u_{h}^{N C}\right) \phi_{i} d x}{\int_{\omega_{i}} \phi_{i} d x}$ for interior nodes $i$, and $f_{i}=0$ otherwise.

\subsection{Upper bound}

We consider first the upper bound of the error and we step the process to the main theorem by the following intermediate lemmas. The first lemma is an adaptation of arguments given in [15], and so the proof will be skipped.

Lemma 2 For interior nodes $i \in \mathcal{N}$, there exists an operator

$$
\Pi_{i}:\left(V\left(\omega_{i}\right)\right)^{d} \longrightarrow\left(\mathcal{P}_{0}^{2}\left(\omega_{i}\right)\right)^{d},
$$

such that for any $v \in V\left(\omega_{i}\right)$ the following conditions hold:

1) For all edge(face) $E \subset \Gamma_{i}, \int_{E}\left(v-\Pi_{i} v\right) \phi_{i} d \gamma=0$.

2) Moreover, $\int_{\omega_{i}}\left(v-\Pi_{i} v\right) \phi_{i} d x=0$, if $x_{i}$ is an interior node.

3) $\left(\int_{\omega_{i}}\left|\nabla \Pi_{i} v\right|^{2} \phi_{i}\right)^{\frac{1}{2}} \leq C\left(\int_{\omega_{i}}|\nabla v|^{2} \phi_{i}\right)^{\frac{1}{2}}$.

where $C$ is a positive constant depending only on the minimum angle of $\mathcal{T}_{h}$.

Lemma 3 For interior nodes $i \in \mathcal{N}$, each $v \in\left(V\left(\omega_{i}\right)\right)^{d}$ and $u_{h}^{N C} \in\left(V_{h}(\Omega)\right)^{d}$, we have

$$
\int_{\omega_{i}} \nabla_{h} u_{h}^{N C}: \nabla\left(\left(\Pi_{i} v\right) \phi_{i}\right) d x=\int_{\omega_{i}} \nabla_{h} u_{h}^{N C}: \nabla\left(v \phi_{i}\right) d x .
$$


Proof. If we denote by $\left[\frac{\partial u_{h}^{N C}}{\partial n_{E}}\right] \in P_{0}(E)$ the jump of the normal derivative across E, we have by applying Green formula and subsequently using the property 1 of lemma 2 ,

$$
\int_{\omega_{i}} \nabla_{h} u_{h}^{N C}: \nabla\left(\left(\Pi_{i} v\right) \phi_{i}\right) d x=\sum_{E \subset \omega_{i}} \int_{E}\left[\frac{\partial u_{h}^{N C}}{\partial n_{E}}\right]\left(\Pi_{i} v\right) \phi_{i} d \gamma=\sum_{E \subset \omega_{i}} \int_{E}\left[\frac{\partial u_{h}^{N C}}{\partial n_{E}}\right] v \phi_{i} d \gamma .
$$

Applying again Green formula yields the result. We give the following lemmas [9]

Lemma 4 There exists a linear operator $I:\left(V_{h}(\Omega)\right)^{d} \longrightarrow\left(V_{h}(\Omega)\right)^{d} \cap\left(H_{0}^{1}(\Omega)\right)^{d}$, satisfying the following estimate.

$$
\begin{aligned}
& \forall u_{h}^{N C} \in V_{h}(\Omega), \forall \omega_{i} \in \mathcal{T}_{h}, k=0,1 \\
& \left\|u_{h}^{N C}-I u_{h}^{N C}\right\|_{k, \omega_{i}} \leq C \sum_{\substack{E \in E_{I} \\
E \cap \omega_{i} \neq \emptyset}} h_{E}^{\frac{1}{2}-k}\left\|\left[u_{h}^{N C}\right]_{E}\right\|_{0, E} .
\end{aligned}
$$

where $h_{E}$ is the diameter of face (edge) $E$.

We have also, the following result:

Lemma 5 For each node $i \in \mathcal{N}$, there exists a linear operator

$$
\Pi_{i}:\left(H_{0}^{1}(\Omega)\right)^{d} \longrightarrow\left(V_{h}(\Omega)\right)^{d},
$$

such that for any $v \in\left(H_{0}^{1}(\Omega)\right)^{d}(\Omega)$ and $\forall T \in \omega_{i} \in \mathcal{T}_{h}$ the following assumptions hold:

1) For all edge $E \subset \Gamma_{i}, \int_{E}\left(v-\Pi_{i} v\right) \phi_{i} d \sigma=0$, and

2) For all edge $s_{h} \in\left(P_{0}(T)\right)^{d \times d}, \int_{\omega_{i}} s_{h} . \nabla\left(v-\Pi_{i} v\right) \phi_{i} d x=0$

3) For all edge $q_{h} \in P_{0}(T), \int_{\omega_{i}} q_{h} \operatorname{div}\left(v-\Pi_{i} v\right) \phi_{i} d x=0$.

The following theorem gives the star-based a posteriori error estimate for the nonconforming finite element approximation of quasi Stokes problem solution.

Theorem 6 There exists a positive constant $C$ depending on the minimum angle of the triangulation such that:

$$
\left(\sum_{i \in \mathcal{N}}\left\|u-u_{h}^{N C}\right\|_{1, \omega_{i}}^{2}\right)^{\frac{1}{2}}+\left\|p-p_{h}\right\|_{0, \Omega} \leq C\left\{\left[\left(\sum_{i \in \mathcal{N}} \eta_{1, i}^{2}+\eta_{2, i}^{2}\right)+\eta_{3}^{2}\right]^{\frac{1}{2}}+o s c(f)\right\} .
$$

where, for more readability, we have skipped the arguments of $\eta_{1, i}, \eta_{2, i}$ and $\eta_{3}$, and so will be done in the sequel.

Proof Since $\left(I u_{h}^{N C}, p_{h}\right) \in\left(H_{0}^{1}(\Omega)\right)^{d} \times L_{0}^{2}(\Omega)$, by standard finite element analysis arguments we state 


$$
\begin{aligned}
\left(\sum_{i \in \mathcal{N}}\left\|u-I u_{h}^{N C}\right\|_{1, \omega_{i}}^{2}\right)^{\frac{1}{2}} & +\left\|p-p_{h}\right\|_{0, \Omega} \\
& \leq C \sup _{\substack{(v, q) \in\left(H_{0}^{1}(\Omega)\right)^{d} \\
\times L_{0}^{2}(\Omega)}} \frac{\left|a((u, p) ;(v, q))-a\left(\left(I u_{h}^{N C}, p_{h}\right) ;(v, q)\right)\right|}{|v|_{1, \Omega}+\|q\|_{0, \Omega}},
\end{aligned}
$$

where $a(. ;$.$) is defined by$

$$
\begin{aligned}
& \forall(u, p),(v, q) \in\left(H_{0}^{1}(\Omega)\right)^{d} \times\left(L_{0}^{2}(\Omega)\right)^{d}, \\
& a((u, p) ;(v, q))=\int_{\Omega} \nabla u . \nabla v d x+\int_{\Omega} \sigma u v d x-\int_{\Omega} p \operatorname{div} v d x+\int_{\Omega} q \operatorname{div} u d x .
\end{aligned}
$$

Then

$$
\begin{aligned}
a((u, p) ;(v, q))-a\left(\left(I u_{h}^{N C}, p_{h}\right) ;(v, q)\right) & =\int_{\Omega}\left(\nabla u-\nabla I u_{h}^{N C}\right): \nabla v d x+\int_{\Omega} \sigma\left(u-I u_{h}^{N C}\right) v d x \\
& -\int_{\Omega}\left(p-p_{h}\right) \operatorname{div} v d x+\int_{\Omega} q \operatorname{div}\left(u-u_{h}^{N C}\right) d x .
\end{aligned}
$$

On one hand, since $\operatorname{div} u=0$ on $\Omega, \sum_{i \in \mathcal{N}} \phi_{i}=1$ and $\phi_{i}$ being bounded we have

$$
\left|\int_{\Omega} q \operatorname{div}\left(u-I u_{h}^{N C}\right) d x\right| \leq C \sum_{\omega_{i} \in \mathcal{T}_{h}}\|q\|_{0, \omega_{i}}\left\|\operatorname{div} I u_{h}^{N C} \phi_{i}^{\frac{1}{2}}\right\|_{0, \omega_{i}} .
$$

By virtue of Lemma 4 with $k=1$ we have

$$
\begin{aligned}
\forall \omega_{i} \in \mathcal{T}_{h},\left\|\operatorname{div} I u_{h}^{N C} \phi_{i}^{\frac{1}{2}}\right\|_{0, \omega_{i}} & \leq\left\|\operatorname{div} u_{h}^{N C} \phi_{i}^{\frac{1}{2}}\right\|_{0, \omega_{i}}+\left\|\left(\operatorname{div} u_{h}^{N C}-\operatorname{div} I u_{h}^{N C}\right) \phi_{i}^{\frac{1}{2}}\right\|_{0, \omega_{i}}, \\
& \leq\left\|\operatorname{div} u_{h}^{N C} \phi_{i}^{\frac{1}{2}}\right\|_{0, \omega_{i}}+C \sum_{E \in E_{I}} h^{-\frac{1}{2}}\left\|\left[u_{h}^{N C}\right]_{E}\right\|_{0, E} .
\end{aligned}
$$

Summing up the contributions, using given indicators definitions and the inequality

$$
\sum_{i} \alpha_{i} \beta_{i} \leq\left(\sum_{i} \alpha_{i}^{2}\right)^{\frac{1}{2}}\left(\sum_{i} \beta_{i}^{2}\right)^{\frac{1}{2}}
$$

we get

$$
\left|\int_{\Omega} q \operatorname{div}\left(u-I u_{h}^{N C}\right) d x\right| \leq C\left(\sum_{i \in \mathcal{N}}\|q\|_{0, \omega_{i}}\right)\left(\sum_{i \in \mathcal{N}} \eta_{1, i}^{2}+\eta_{3}^{2}\right)^{\frac{1}{2}}
$$

On the other hand, let $v$ be an element of $\left(H_{0}^{1}(\Omega)\right)^{d}$ and set $\widetilde{v}:=\sum_{i \in \mathcal{N}} v_{i} \phi_{i}$, where $v_{i}=\frac{\int_{\omega_{i}} v \phi_{i} d x}{\int_{\omega_{i}} \phi_{i} d x}$ for interior nodes and 0 otherwise. 


$$
\begin{aligned}
A= & \int_{\Omega}\left(\nabla u-\nabla I u_{h}^{N C}\right): \nabla v d x+\int_{\Omega} \sigma\left(u-I u_{h}^{N C}\right) v d x-\int_{\Omega}\left(p-p_{h}\right) \operatorname{div} v d x \\
= & \sum_{\omega_{i} \in \mathcal{T}_{h}} \int_{\omega_{i}}\left(\nabla u-\nabla u_{h}^{N C}\right): \nabla v d x+\int_{\omega_{i}}\left(\nabla u_{h}^{N C}-\nabla I u_{h}^{N C}\right): \nabla v d x \\
& +\int_{\Omega} \sigma\left(u-u_{h}^{N C}\right) v d x-\int_{\omega_{i}}\left(p-p_{h}\right) \operatorname{div} v d x+\int_{\Omega} \sigma\left(u_{h}^{N C}-I u_{h}^{N C}\right) v d x \\
= & -\sum_{\omega_{i} \in \mathcal{T}_{h}} \int_{\omega_{i}}\left(\nabla u_{h}^{N C}\right): \nabla v d x-\int_{\omega_{i}} \sigma u_{h}^{N C} v d x+\int_{\omega_{i}} p_{h} \operatorname{div} v d x+\int_{\omega_{i}} f v d x \\
& +\sum_{\omega_{i} \in \mathcal{T}_{h}} \int_{\omega_{i}}\left(\nabla u_{h}^{N C}-\nabla I u_{h}^{N C}\right) \cdot \nabla v d x+\int_{\Omega} \sigma\left(u_{h}^{N C}-I u_{h}^{N C}\right) v d x .
\end{aligned}
$$

Knowing that $\widetilde{v} \in\left(V_{h}(\Omega)\right)^{d} \cap\left(H_{0}^{1}(\Omega)\right)^{d}$,

$$
\begin{aligned}
A= & -\sum_{\omega_{i} \in \mathcal{T}_{h}} \int_{\omega_{i}}\left(\nabla u_{h}^{N C}\right): \nabla(v-\widetilde{v}) d x-\int_{\omega_{i}} \sigma u_{h}^{N C}(v-\widetilde{v}) d x \\
& +\int_{\omega_{i}} p_{h} \operatorname{div}(v-\widetilde{v}) d x+\int_{\omega_{i}} f(v-\widetilde{v}) d x \\
& +\sum_{\omega_{i} \in \mathcal{T}_{h}} \int_{\omega_{i}}\left(\nabla u_{h}^{N C}-\nabla I u_{h}^{N C}\right): \nabla v d x+\int_{\Omega} \sigma\left(u_{h}^{N C}-I u_{h}^{N C}\right)(v-\widetilde{v}) d x .
\end{aligned}
$$

Stating that $v-\widetilde{v}=\sum_{i \in \mathcal{N}}\left(v-v_{i}\right) \phi_{i}$, and using $\sum_{i \in \mathcal{N}} \phi_{i}=1$.

Since $\left(v-v_{i}\right) \in\left(V\left(\omega_{i}\right)\right)^{d}$, adding and removing same quantities in the two last terms and use item 2 of Lemma 5 we obtain

$$
\begin{aligned}
A= & -\sum_{\omega_{i} \in \mathcal{T}_{h}} \int_{\omega_{i}}\left(\nabla u_{h}^{N C}\right): \nabla \Pi\left(v-v_{i}\right) \phi_{i} d x-\int_{\omega_{i}} \sigma u_{h}^{N C} \Pi\left(v-v_{i}\right) \phi_{i} d x \\
& +\int_{\omega_{i}} p_{h} \operatorname{div} \Pi\left(v-v_{i}\right) \phi_{i} d x+\int_{\omega_{i}} f \Pi\left(v-v_{i}\right) \phi_{i} d x \\
& -\int_{\omega_{i}} \sigma u_{h}^{N C}\left(v-v_{i}-\Pi\left(v-v_{i}\right)\right) \phi_{i} d x+\int_{\omega_{i}} p_{h} \operatorname{div}\left(v-v_{i}-\Pi\left(v-v_{i}\right)\right) \phi_{i} d x \\
& +\sum_{\omega_{i} \in \mathcal{T}_{h}} \int_{\omega_{i}}\left(\nabla u_{h}^{N C}-\nabla I u_{h}^{N C}\right): \nabla v d x+\int_{\omega_{i}} f\left(v-v_{i}-\Pi\left(v-v_{i}\right)\right) \phi_{i} d x .
\end{aligned}
$$

Finally, using the definition of local problems $\left(S P_{i}\right)$,

$$
\begin{aligned}
A= & -\sum_{\omega_{i} \in \mathcal{T}_{h}} \int_{\omega_{i}}\left(\nabla \eta_{i}\right): \nabla\left(v-v_{i}\right) \phi_{i} d x-\int_{\omega_{i}} \sigma u_{h}^{N C}\left(v-v_{i}-\Pi\left(v-v_{i}\right)\right) \phi_{i} d x \\
& +\int_{\omega_{i}} p_{h} \operatorname{div}\left(v-v_{i}-\Pi\left(v-v_{i}\right)\right) \phi_{i} d x \\
& +\sum_{\omega_{i} \in \mathcal{T}_{h}} \int_{\omega_{i}}\left(\nabla u_{h}^{N C}-\nabla I u_{h}^{N C}\right): \nabla v d x+\int_{\omega_{i}} f\left(v-v_{i}-\Pi\left(v-v_{i}\right)\right) \phi_{i} d x .
\end{aligned}
$$


We now process successively with each term of the right-hand side. On one hand, using Cauchy-Schwarz and item 2 of Lemma 2 we have

$$
\begin{aligned}
\sum_{i \in \mathcal{N}}\left[\int_{\omega_{i}} \nabla \eta_{i}: \nabla \Pi_{i}\left(v-v_{i}\right) \phi_{i} d x\right] & \leq\left(\sum_{i \in \mathcal{N}} \int_{\omega_{i}}\left|\nabla \eta_{i}\right|^{2} \phi_{i} d x\right)^{\frac{1}{2}}\left(\sum_{i \in \mathcal{N}} \int_{\omega_{i}}\left|\nabla \Pi_{i}\left(v-v_{i}\right)\right|^{2} \phi_{i} d x\right)^{\frac{1}{2}} \\
& \leq C\left(\sum_{i \in \mathcal{N}} \eta_{2, i}^{2}\left(u_{h}^{N C}, p_{h}\right)\right)^{\frac{1}{2}}\left(\sum_{i \in \mathcal{N}} \int_{\omega_{i}}\left|\nabla\left(v-v_{i}\right)\right|^{2} \phi_{i} d x\right)^{\frac{1}{2}} \\
& \leq C\left(\sum_{i \in \mathcal{N}} \eta_{2, i}^{2}\left(u_{h}^{N C}, p_{h}\right)\right)^{\frac{1}{2}}\|v\|_{1, \Omega}
\end{aligned}
$$

on the other hand, since both of $\left(v-v_{i}\right)$ and $\Pi_{i}\left(v-v_{i}\right)$ belong to $V\left(w_{i}\right)$, using definition of $V\left(w_{i}\right)$ and coefficients $f_{i}$ give

$$
\int_{\omega_{i}}\left(f-\sigma u_{h}^{N C}\right)\left(v-v_{i}-\Pi_{i}\left(v-v_{i}\right)\right) \phi_{i} d x=\int_{\omega_{i}}\left(f-f_{i}-\sigma u_{h}^{N C}\right)\left(v-v_{i}-\Pi_{i}\left(v-v_{i}\right)\right) \phi_{i} d x
$$

for any constant $f_{i} \in \mathbf{R}$ for all interior nodes. Defining $f_{i}=0$ for boundary nodes, we have

$$
\begin{aligned}
\int_{\omega_{i}}\left(f-\sigma u_{h}^{N C}\right)\left(v-v_{i}-\Pi_{i}\left(v-v_{i}\right)\right) \phi_{i} d x \leq & \left\|\left(f-f_{i}\right)\left(\phi_{i}\right)^{\frac{1}{2}}\right\|_{0, \omega_{i}}\left(\left\|\left(v-v_{i}\right)\left(\phi_{i}\right)^{\frac{1}{2}}\right\|_{0, \omega_{i}}\right. \\
& \left.+\left\|\left(\Pi_{i}\left(v-v_{i}\right)\right)\left(\phi_{i}\right)^{\frac{1}{2}}\right\|_{0, \omega_{i}}\right),
\end{aligned}
$$

for every nodes. Using Cauchy-Schwarz then the inequality (1) and once more $\sum_{i \in \mathcal{N}} \phi_{i}=1$ we get

$$
\begin{aligned}
\sum_{i \in \mathcal{N}} & {\left[\int_{\omega_{i}}\left(f-\sigma u_{h}^{N C}\right)\left(v-v_{i}-\Pi_{i}\left(v-v_{i}\right)\right) \phi_{i} d x\right] } \\
\leq \quad & C\left(\sum_{i \in \mathcal{N}}\left\|h_{i}\left(f-f_{i}\right)\left(\phi_{i}\right)^{\frac{1}{2}}\right\|^{2}\right)^{\frac{1}{2}}\left(\sum _ { i \in \mathcal { N } } h _ { i } ^ { - 2 } \left(\left\|\left(v-v_{i}\right)\left(\phi_{i}\right)^{\frac{1}{2}}\right\|_{0, \omega_{i}}\right.\right. \\
& \left.\left.+\left\|\left(\Pi_{i}\left(v-v_{i}\right)\right)\left(\phi_{i}\right)^{\frac{1}{2}}\right\|_{0, \omega_{i}}\right)\right), \\
\leq \quad & \quad \operatorname{Cosc}(f)\|v\|_{1, \Omega},
\end{aligned}
$$

where $C$ is a generic constant only depending on the minimum angle of triangulation.

Using successively item 3. of Lemma 5 and Lemma 4 with $k=1$ we get

$$
A \leq\left\{\left(\sum_{i \in \mathcal{N}} \eta_{2, i}^{2}+\eta_{3}^{2}\right)^{\frac{1}{2}}+o s c(f)\right\}|v|_{1, \Omega}
$$

Summing up the contributions gives,

$$
\begin{aligned}
& \left|a((u, p) ;(v, q))-a\left(\left(I u_{h}^{N C}, p_{h}\right) ;(v, q)\right)\right| \\
& \left.\leq\left(\sum_{i \in \mathcal{N}}\|q\|_{0, \omega_{i}}\right)\left(\sum_{i \in \mathcal{N}} \eta_{1, i}^{2}+\eta_{3}^{2}\right)^{\frac{1}{2}}+\left\{\eta_{2, i}^{2}+\eta_{3}^{2}\right\}^{\frac{1}{2}}+o s c(f)\right\}|v|_{1, \Omega},
\end{aligned}
$$


which finally gives us:

$$
\begin{aligned}
\sum_{i \in \mathcal{N}}\left\|u-u_{h}^{N C}\right\|_{1, \omega_{i}}^{2} & \leq \sum_{i \in \mathcal{N}}\left\|u-I u_{h}^{N C}\right\|_{1, \omega_{i}}^{2}+\sum_{i \in \mathcal{N}}\left\|u_{h}^{N C}-I u_{h}^{N C}\right\|_{1, \omega_{i}}^{2} \\
& \leq C \eta_{3}^{2}+\sum_{i \in \mathcal{N}}\left\|u-I u_{h}^{N C}\right\|_{1, \omega_{i}}^{2} .
\end{aligned}
$$

and yields the result.

\subsection{Efficiency of the estimator}

This section is devoted to the estimator efficiency. Hence, we first need the following theorem:

Theorem 7 There exists a positive constant $C$ depending on the minimum angle of the triangulation such that $: \forall \omega_{i} \in \mathcal{T}_{h}$, we have

$$
\begin{gathered}
\left\|\operatorname{div}_{h}^{N C}\right\|_{0, \omega_{i}} \leq C\left\|u-u_{h}^{N C}\right\|_{1, \omega_{i}}, \\
\eta_{2} \leq C\left\|u-u_{h}^{N C}\right\|_{1, \omega_{i}}+\left\|p-p_{h}\right\|_{0, \omega_{i}},
\end{gathered}
$$

and

$$
\eta_{3} \leq C\left\|u-u_{h}^{N C}\right\|_{1, \omega_{i}}
$$

Proof. It is clear that:

$$
\left.\left\|\operatorname{div} u_{h}^{N C} \phi_{i}^{\frac{1}{2}}\right\|_{0, \omega_{i}}\right) \leq C\left\|u-u_{h}^{N C}\right\|_{1, \omega_{i}} .
$$

For each $i \in \mathcal{N}$, by definition of $\eta_{2, i}\left(u_{h}^{N C}\right)$ and taking test function $\mu_{i}=\eta_{i}$ in local problem $\left(S P_{i}\right)$ give

$$
\begin{aligned}
\eta_{2, i}^{2}\left(u_{h}^{N C}, p_{h}\right) & =\int_{\omega_{i}}\left(\left|\nabla \eta_{i}\right|^{2} \phi_{i} d x\right) \\
& =\int_{\omega_{i}} \nabla u_{h}^{N C}: \nabla\left(\eta_{i} \phi_{i}\right) d x+\int_{\omega_{i}}\left(\sigma u_{h}^{N C} \eta_{i}\right) \cdot \phi_{i} d x-\int_{\omega_{i}}\left(p_{h} \operatorname{div}\left(\eta_{i}\right) \phi_{i}\right) d x-\int_{\omega_{i}} f \eta_{i} \phi_{i} d x .
\end{aligned}
$$

Since $\left(\eta_{i} \phi_{i}\right) \in\left(H_{0}^{1}(\Omega)\right)^{d}$, we can state

$$
\begin{aligned}
\eta_{2, i}^{2}\left(u_{h}^{N C}, p_{h}\right)= & \int_{\omega_{i}}\left(\nabla u_{h}^{N C}-\nabla u\right): \nabla\left(\eta_{i} \phi_{i}\right) d x+\int_{\Omega} \sigma\left(u_{h}^{N C}-u\right) \cdot \eta_{i} \phi_{i} d x-\int_{\omega_{i}}\left(p_{h}-p\right) \operatorname{div}\left(\eta_{i} \phi_{i}\right) d x, \\
= & \int_{\omega_{i}}\left(\nabla u_{h}^{N C}-\nabla u\right): \nabla\left(\eta_{i}\right) \phi_{i} d x+\int_{\omega_{i}}\left(\nabla u_{h}^{N C}-\nabla u\right)\left(\eta_{i}\right) \cdot \nabla\left(\phi_{i}\right) d x \\
& +\int_{\Omega} \sigma\left(u_{h}^{N C}-u\right) \eta_{i} \phi_{i} d x-\int_{\omega_{i}}\left(p_{h}-p\right) \operatorname{div}\left(\eta_{i} \phi_{i}\right) d x .
\end{aligned}
$$

Applying Cauchy-Schwarz inequality gives

$$
\begin{aligned}
\eta_{2, i}^{2}\left(u_{h}^{N C}, p_{h}\right) \leq & \left\|u-u_{h}^{N C}\right\|_{1, \omega_{i}} \eta_{2, i}\left(u_{h}^{N C}, p_{h}\right)+\left\|u-u_{h}^{N C}\right\| 1, \omega_{i}\left\|\eta_{i}\right\|_{0, \omega_{i}}\|\phi\|_{W^{1, \infty}\left(\omega_{i}\right)} \\
& +\|\sigma\|_{0, \infty}\left\|u-u_{h}^{N C}\right\|_{0, \omega_{i}}\left\|\eta_{i}\right\|_{L^{\infty}\left(\omega_{i}\right)}+\left\|p-p_{h}\right\|_{0, \omega_{i}} \eta_{2, i}\left(u_{h}^{N C}, p_{h}\right),[5]
\end{aligned}
$$


where $\phi$ is bounded in $\omega_{i}$. Now since $\eta_{i} \in V\left(\omega_{i}\right)$, using (1), we have

$$
\left\|\eta_{i}\right\|_{0, \omega_{i}} \leq C h_{i} \eta_{(2, i)}\left(u_{h}^{N C}, p_{h}\right) .
$$

Finally using the property $\left|\phi_{i}\right|_{W^{1, \infty}\left(\omega_{i}\right)} \leq \frac{C}{h_{i}}$, we get

$$
\eta_{2, i}\left(u_{h}^{N C}, p_{h}\right) \leq C\left(1+\|\sigma\|_{L^{\infty}\left(\omega_{i}\right)} h_{i}\right)\left\|u-u_{h}^{N C}\right\|_{1, \omega_{i}}+\left\|p-p_{h}\right\|_{0, \omega_{i}} .
$$

We refer for a proof of third estimate to [9], which concludes the proof.

\section{Mesh adaption procedure and Numerical results.}

For the numerical illustration of the efficiency of the error estimator and the based adaption process, we consider tree examples for different singularity geometry.

\subsection{Example 1.}

In this example, we consider a simple problem with a homogeneous data on the computational domain $[0,1]^{2}$, as shown in figure 1 , with the source term $f$ given by the exact solution $u=\left[u_{1}, u_{2}\right]$ and the pressure $p$.

$$
\begin{gathered}
u_{1}=250000 x^{4} y^{3}(1-y)^{5}\left(4\left(1-5 y+9 y^{2}-7 y^{3}+2 y^{4}\right)-5 y+24 y^{2}-42 y^{3}+32 y^{4}-9 y^{5}\right)+0.3 x \\
u_{2}=250000 y^{4} x^{3}(1-x)^{5}\left(4\left(1-5 x+9 x^{2}-7 x^{3}+2 x^{4}\right)-5 x+24 x^{2}-42 x^{3}+32 x^{4}-9 x^{5}\right)+0.3 y \\
p_{1}=2510^{4} y^{4} x^{3}(1-y)^{5}\left(4-20 x+36 x^{2}-28 x^{3}+8 x^{4}-5 x+24 x^{2}-42 x^{3}+32 x^{4}-9 x^{5}\right) \\
p_{2}=\left(4\left(1-5 y+9 y^{2}-7 y^{3}+2 y^{4}\right)-5 y+24 y^{2}-42 y^{3}+32 y^{4}-9 y^{5}\right) \\
p=p_{1} p_{2}
\end{gathered}
$$

We perform nonconforming finite element discretization on it, and we report on Figures $1,2,3$ and 4 a sequence of adapted meshes using the proposed refinement indicators and corresponding computed velocity and pressure respectively. 


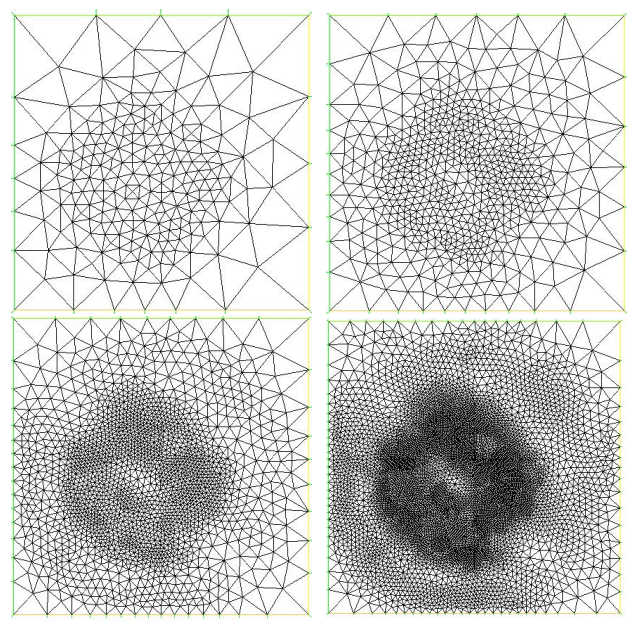

Figure 1. Adaptively refined mesh after 1 (top left), 3 (top right), 6 (bottom left), and 9 (bottom right) refinement steps.
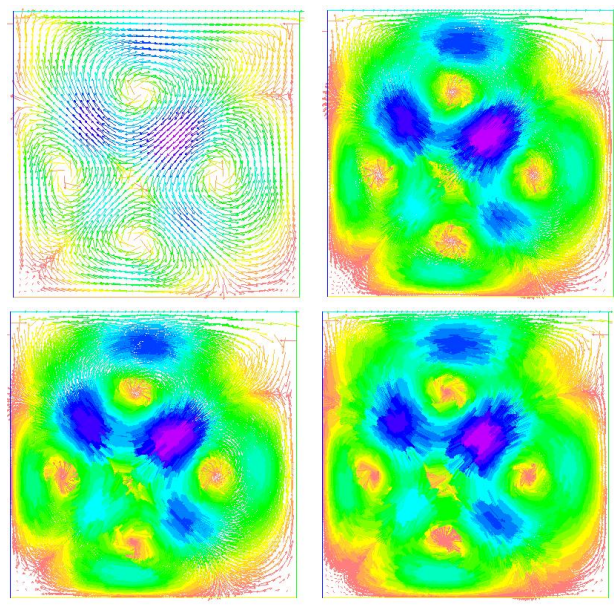

Figure 2. The Velocity distribution corresponds to the meshes shown in figure 1. 
58 A R I M A - Volume 16-2013
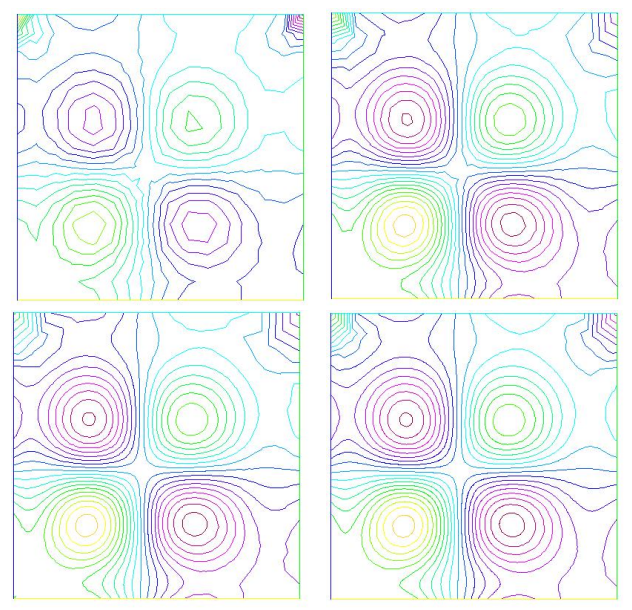

Figure 3. The pressure distribution corresponds to the meshes shown in figure 1.
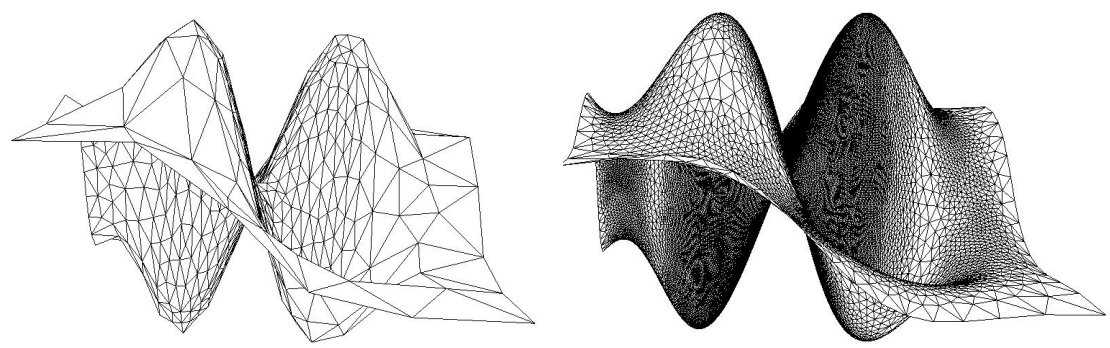

Figure 4. approximate pressure.

On the left, the first iteration with 467 DOF, on the right the last iteration with 5667 DOF.

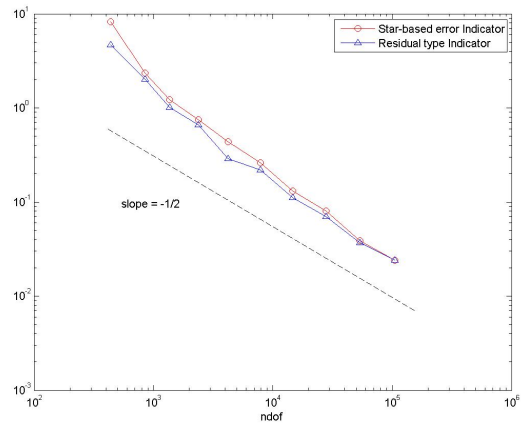

Figure 5. Evolution of residual error indicator and star-based error indicator for quasiStokes example.

In figure 5, we have included the error indicator behavior against the number of degree of freedom. Let us remark that the error decreases in energy norm as the number of DOF increases, compared with a slope $-\frac{1}{2}$. 


\section{A comparison with residual type estimator:}

The new estimator is compared now with the residual type estimator [16]. We will take the same quasi-Stokes example as above. Successive iterations of adapted mesh are represented in Figure 6 for the residual type estimator, and Figure 5 gives comparison of asymptotic decays of the two estimators versus the number of degrees of freedom (ndof). We observe analogous behavior of the two estimators for this example.
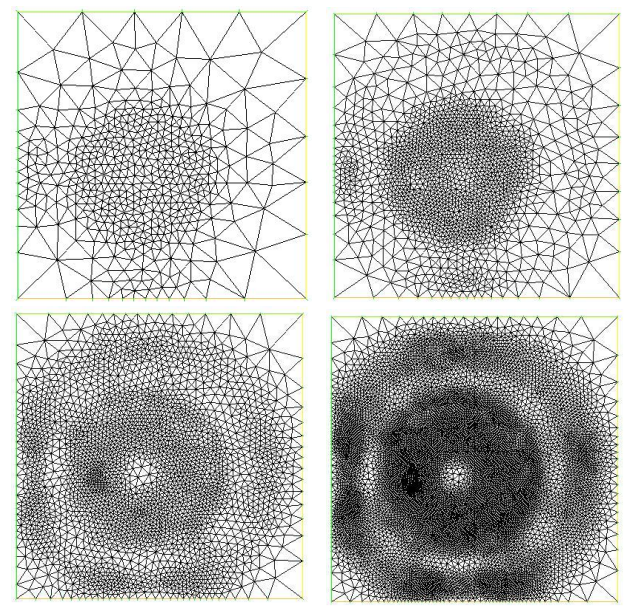

Figure 6. Adaptively refined mesh after 1 (top left), 3 (top right), 6 (bottom left), and 9 (bottom right) refinement steps.

\subsection{Example 2, Backward facing Step.}

In this example, we consider the Backward facing Step. Here, the spikes in the pressure are noted due to a discontinuity in the boundary conditions, but to a discontinuity in the geometry of the boundary. The figures 7, 8 and 9 show the adaptive meshes, corresponding velocity and pressure elevation [10]. 
60 A R I M A - Volume $16-2013$
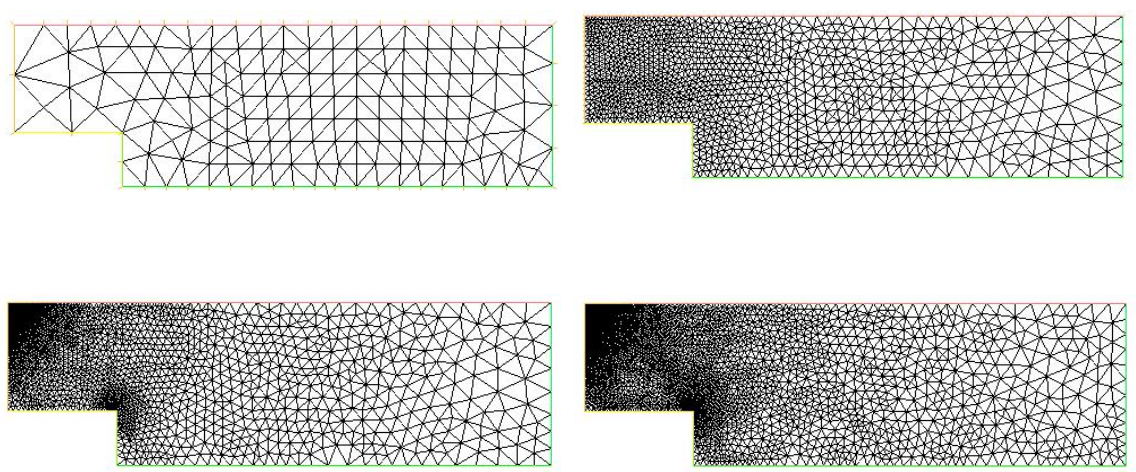

Figure 7. Adaptively refined mesh after 1 (top left), 3 (top right), 6 (bottom left), and 9 (bottom right) refinement steps.
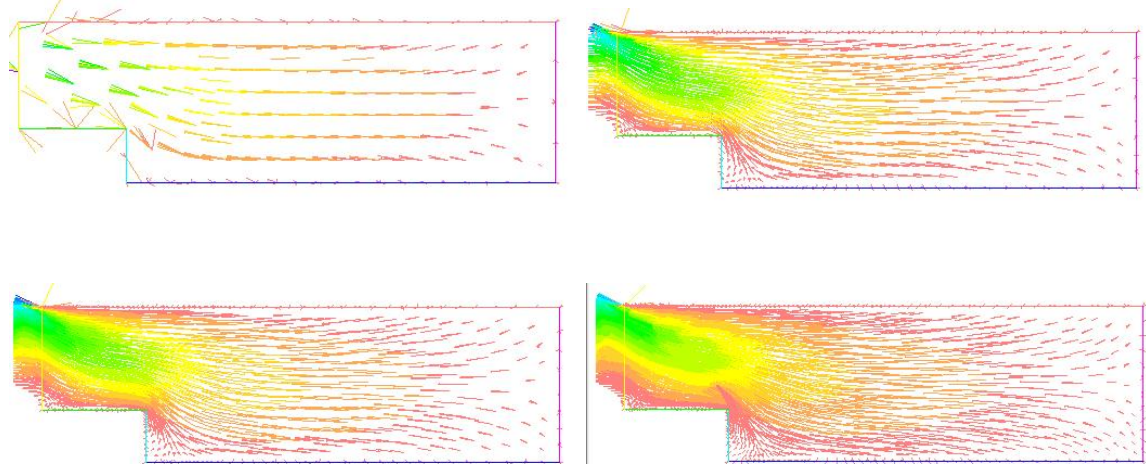

Figure 8. The velocity distribution corresponds to the meshes shown in figure 7.

On the left the pressure on the uniform grid, on the right the adapted grid . 


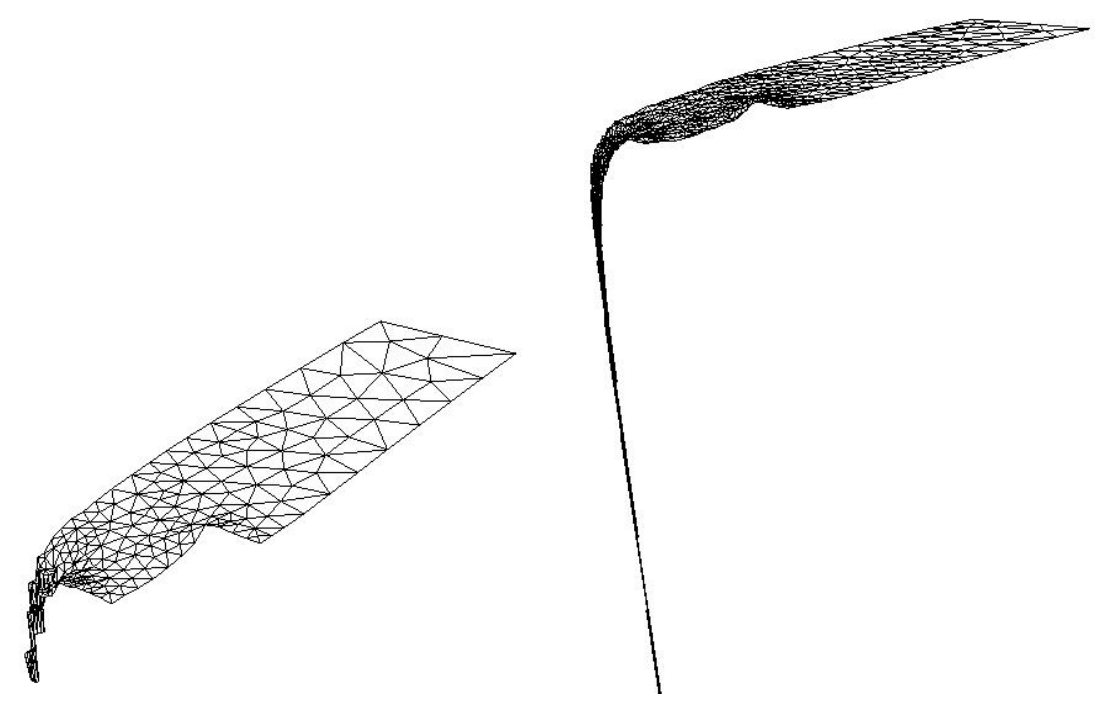

Figure 9. approximate pressure.

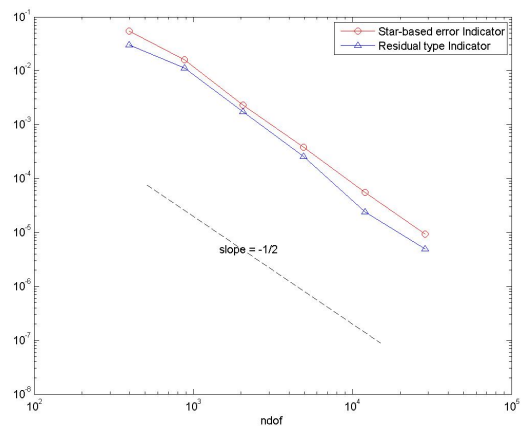

Figure 10. Evolution of residual error indicator and star-based error indicator for quasiStokes example.

The initial grid is uniformly refined, 4 levels of refinement, or adaptively refined based on the error estimate computed from the solution. In the last case triangles were added near the re-entrant corner to resolve the discontinuity, profiles of the velocity field are very similar this time but the drop in pressure is better represented with the adaptive strategy done near the boundary.

In figure 10, we have included the error indicator behavior against to the number of degree of freedom, compared with a slope $-\frac{1}{2}$, and gives a comparison with the residual type estimators. 
62 A R I M A - Volume 16 - 2013

\subsection{Example 3: Disk with a Crack.}

Finally, we test our error estimate on a quasi Stokes flow in a disk of radius 1 with a crack joining the center to the boundary [10].

- The right-hand side $f$ is 0 .

- The boundary conditions are $u^{t}=\frac{3}{2}\left(\cos \frac{\theta}{2}-\cos \frac{3 \theta}{2}, 3 \sin \frac{\theta}{2}-\sin \frac{3 \theta}{2}\right)$.

- $(r, \theta)$ is a polar representation of a point in the disk.

- The exact solution is:

$$
u^{t}=\frac{3}{2} \sqrt{r}\left(\cos \frac{\theta}{2}-\cos \frac{3 \theta}{2}, 3 \sin \frac{\theta}{2}-\sin \frac{3 \theta}{2}\right) \text {. and } p=\frac{6}{\sqrt{r}} \cos \frac{\theta}{2} .
$$
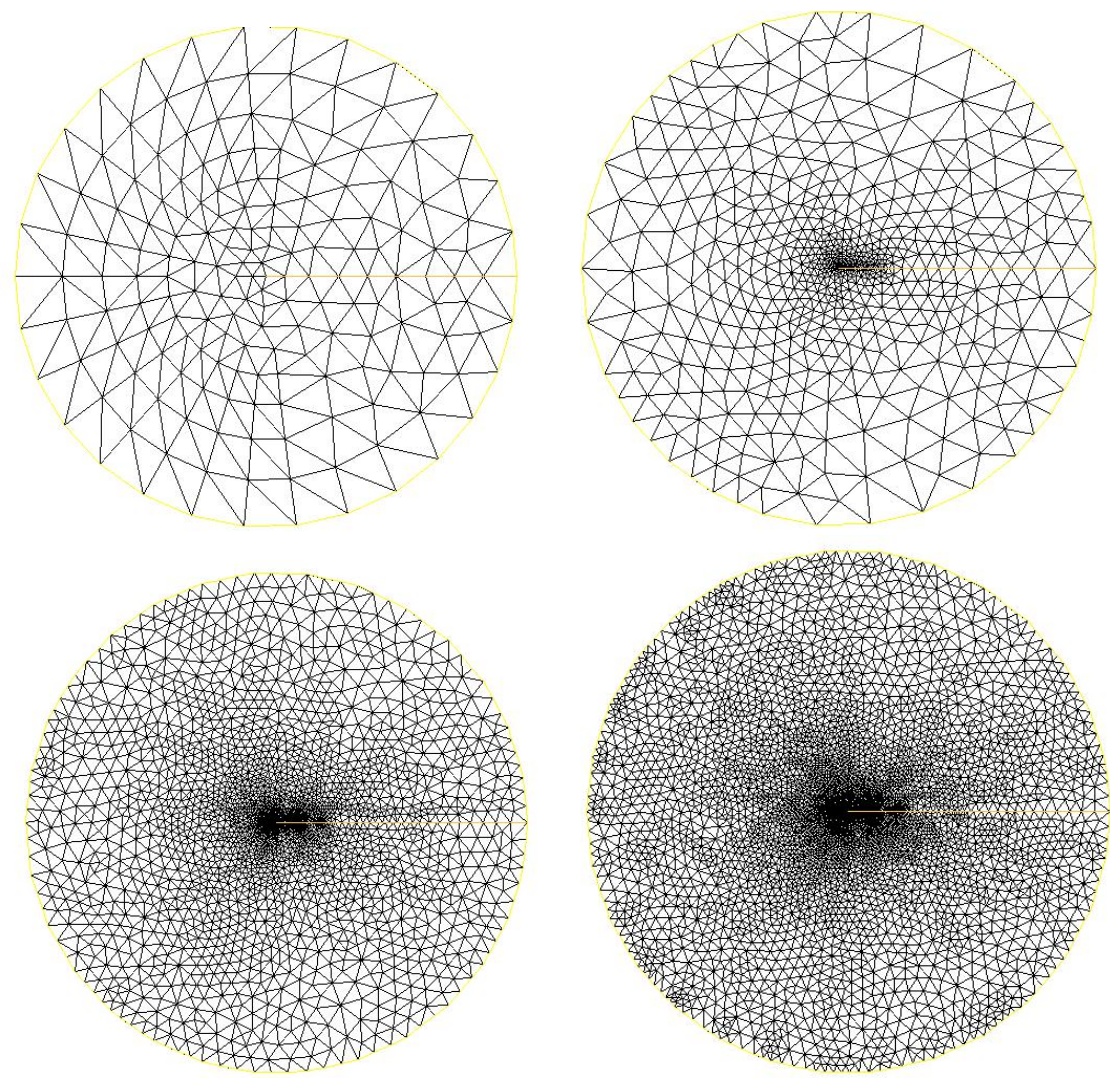

Figure 11. Adaptively refined mesh after 1 (top left), 3 (top right), 6 (bottom left), and 9 (bottom right) refinement steps. 

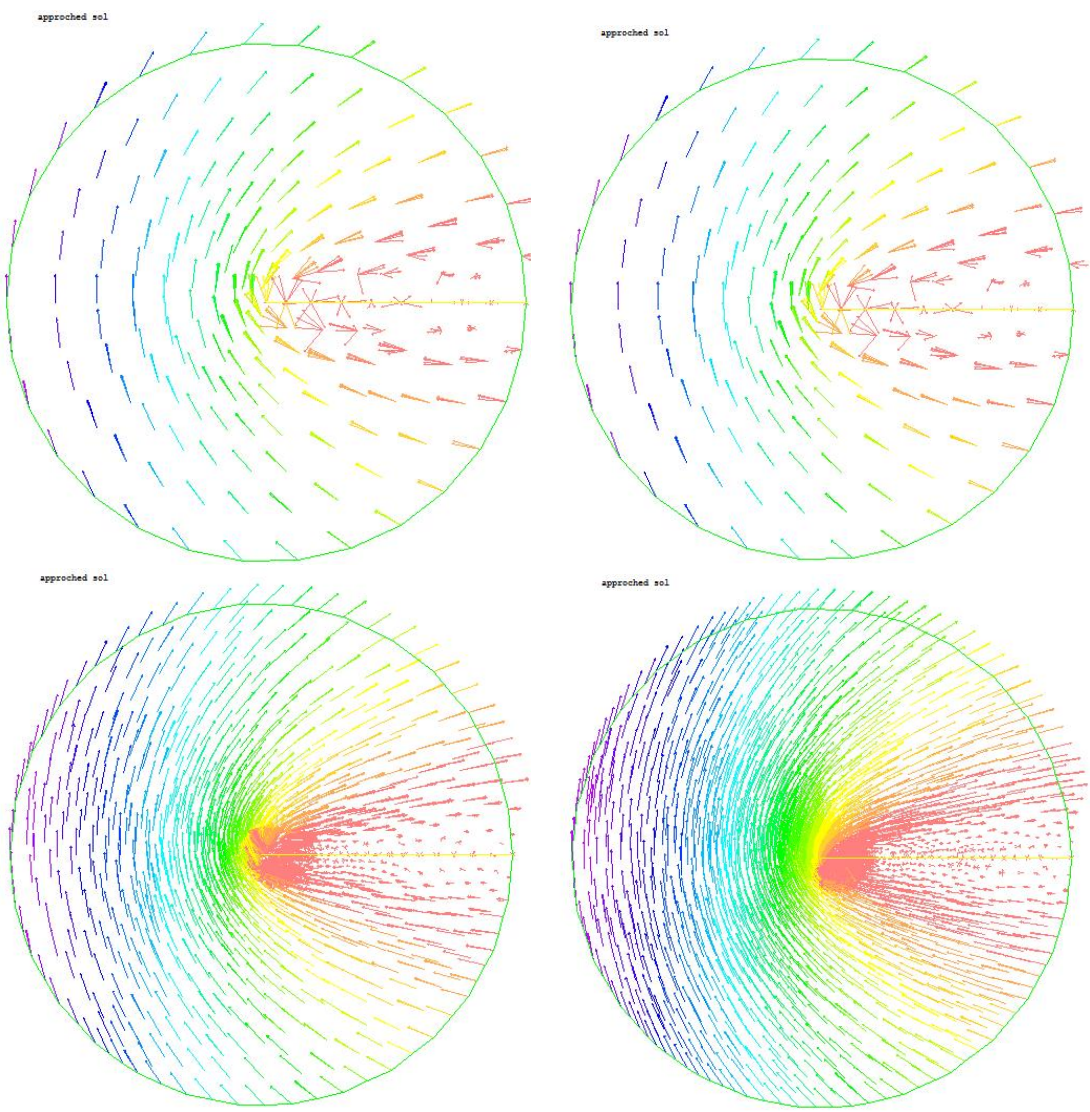

Figure 12. The velocity distribution corresponds to the meshes shown in figure . 
64 A R I M A - Volume $16-2013$
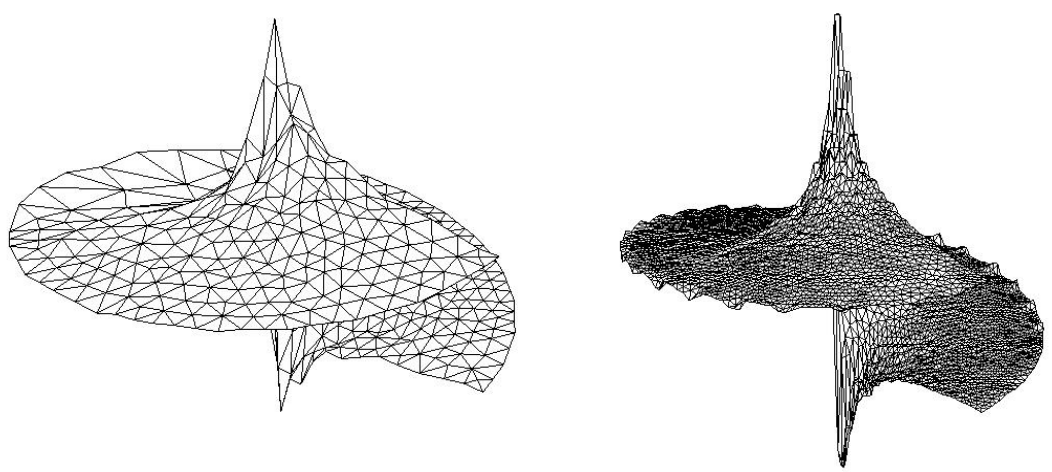

Figure 13. approximate pressure.

On the left the pressure on the uniform grid on the right the adapted grid.

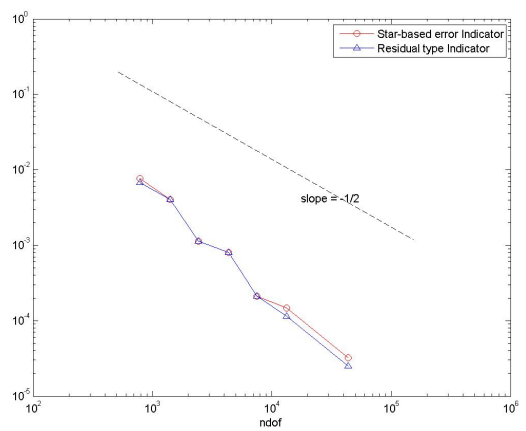

Figure 14. Evolution of residual error indicator and star-based error indicator for quasiStokes example.

The solution was computed on each grid, along with error estimates, during the refinement process, (Figures 11, 12, 13 and 14), these estimates were computed using an interpolation scheme for the values at the new nodes, consequently their accuracy deteriorates along with the number of refinement steps. However, we used intermediate recalculations based on the computation of the estimates, we would have a mesh with more levels of refinement around the singularity(hence giving higher level for the pressure). In that regard, the use of interpolated values instead of computed solution values had a grid smoothing effect, and the figure 14 gives a comparison with the residual type estimators.

\section{Conclusion}

In this work, we have presented an a posteriori error analysis for a nonconforming finite element method to approximate the quasi Stokes problem. The a posteriori error analysis of this scheme give the global upper bound and local lower bound. The perspec- 
tive of this work is to adapt this technique to the Navier Stokes problem and the evolution Stokes problem.

\section{Acknowledgement}

The authors would like to thank the reviewers for the thoughtful comments on our paper. This work is supported in part by HydroMed Project, and CNRST (Projet d'établissement, Université Hassan $1^{\text {er }}$ Settat, Ministère de l'enseignement supérieur, Maroc). Volubilis Projects ( PHC M.A/13/286) and (PHC MA/11/246).

These numerical simulations have been carried out using the software FreeFem++ developed by Frédéric Hecht : http://www.freefem.org/.

\section{References}

[1] B. Achchab, A. Agouzal, N. Debit, K. Bouihat, "Star-based a posteriori error estimators for elliptic problems”, (TO APPEAR IN J. SCI. COMPUT.).

[2] B. AchChab, A. Agouzal, J. Baranger, J.F. Maitre, " Estimateur d'erreur a posteriori hiérarchique. Application aux éléments finis mixtes.”, NUMER. MATH., VOL. (80), 159-179, 1998.

[3] B. Aсhсhab, A. Agouzal, A. El Fatini, A. Souissi, "Robust hierarchical a posteriori error estimates for stabilized convection-diffusion problem”, NUMER. MATH. PART. DifF. EQU. VOLUME 28, ISSUE 5, 1717-1728, 2012.

[4] B. Aсhсhав, M. El Fatini, A. Ern, A. Souissi, " A posteriori error estimates for subgrid viscosity stabilized approximations of convection-diffusion equations ,", APPL. MATH. LETT, VOL 22 ISSUE 9,1418-1424, 2009.

[5] A. Agouzal, " A posteriori error estimator for nonconforming finite element methods.", APPL. MATH. LETT, 7(5):1017-1033, 1994.

[6] M. Ainsworth, I. BABÛSKA, " Reliable and robust a posteriori error estimating for singularly perturbed reaction-diffusion problems,", SIAM J. NUMER. ANAL. 36, 1999.

[7] A. Alonso, "Error estimators for a mixed method", Numer. MATH, 74, 385-395, 1996.

[8] R.E. BANK AND A. WEISER, " Some a posteriori error estimators for elliptic partial differential equations,", MATH. COMP. V 44, 283-301. 1985.

[9] O. A. KARAKASHIAN AND F. PASCAL, “ A posteriori error estimates for discontinuous Galerkin approximation of second-order elliptic problems. ", SIAM J. NUM ANAL. 41, 6, 2374-2399, 2003.

[10] R.E. BAnK AND D. B. Welfert, “ A posteriori error estimates for the Stokes problem,”, SIAM JOURNAL ON NUMERICAL ANALYSIS. V 28, PP 591-623. 1991.

[11] D. BRAESS AND R. VERFÜRTH, " Error estimators for a the Raviart-Thomas element”, SIAM NUMER. ANAL, 33, 2431-2444, 1996.

[12] C. CARstensen. "A posteriori error estimate for a mixed finite element method", MATH. COMP. 66, 465-476. 1997.

[13] E. DARI R. DURÁN AND C PADRA, “ Error estimators for nonconforming finite element approximations of the Stokes problem ”, МАTH. COMP. VOL 64. N 211, 1017-1033. 1995. 
66 A R I M A - Volume 16-2013

[14] R. H. W. Hoppe, et B. I. Wohlmulth, “ A comparaison of a posteriori error estimators for mixed finite element discretizations.”, $M^{2} A N$, vOL 30, $n$ 2, 237-263, 1996.

[15] P. Morin, R.H. Nochetto And K.G. Siebert, " Local Problems on Stars: A Posteriori Error Estimators, Convergence, and Performance.”, MATH. COMP. VOL 72, 1067-1097. 2003.

[16] R. VERFÜRTH. “ A review of a posteriori error estimation and adaptive mesh-refinement techniques.” Teubner SKriPten ZUR Numerik, B.G. Teubner Stuttgart 1996. 